\title{
Apply with WASP Water Quality Model
}

\author{
Hao Lifen \\ Engineering college of Shanxi University \\ Taiyuan, China \\ Bi Xingwen \\ Taiyuan IRON\&STEEL(GROUP) CO.,LTD. \\ Taiyuan, China
}

\author{
Cheng Lianbiao \\ JINGWEI Textile Machinery CO.,LTD. \\ Taiyuan, China
}

\begin{abstract}
WASP(The water quality analysis simulation program) was recommended by EPA used as water quality model. It has been applied widely, It can simulate steady or unsteady water quality processes in such diverse water bodies as streams, lakes, reservoirs, estuaries. This paper introduced the compositions (DYNHYD、EUTRO、TOXI), and the trans -formations among 8 targets in EUTRO model, its applications in and aboard, and its develop mental prospects and directions in future.
\end{abstract}

Keywords- WASP, water quality simulation, apply

\section{INTRODUCTION}

The model studies natural water to influence to enter water in the nature or mankind's activity quality to change mathematics description of regulation with time and space. Involve hydrology, water power, water chemistry, water living creature, mathematics, and calculator...etc. several academics knowledge, direct evaluate for fluid matter, estimate and pollution adjust to control with management provide basis[1].

WASP(The water quality analysis simulation program) is developed by the environmental protection bureau environment research laboratory in the United States nation, can use to imitate hydrology dynamics, river one dimension unsteady flow, lake and river mouth Be 3D unsteady to flow, normal regulations pollutant(include to fuse oxygen, living creature oxygen consumption and nourishment material and seaweed pollution) and poisonous pollutant(include organic chemistry material, metal and deposition thing the migration and conversion regulation in the water. The WASP fluid matter model mainly has three functions: describe fluid matter present condition, provide a general fluid matter estimate and provide particular position fluid matter estimate.

The edition of the most original WASP is release in 1983[2], it synthesized the concept that many other models uses, after several emendations, gradually become one of the models of the development maturity of USEPA.WASP5 and it one-time editions are all DOS procedures[3-4], but WASP6 development is under Window of procedure[5].Developing in 2005 can under the Windows2000 and the XP system the WASP7 editionses of movement.

WASP6 and WASP7s all have visual operation interface, circulating the speed is that 10 times that of the DOS edition in past is above[6]. Their main characteristicses are: Develop friendly customer's interface according to Windows; Including can convert born WASP can identify of processing data format; Have to efficiently enrich nourishment to turn and the processing mold of the organic pollutant piece;The result computing a result and actually measuring can directly carry on a curve comparison[7].

\section{WASP MODEL}

WASP includes two independent calculation procedures: DYNHYD and WASP. They can unite movement or independently circulate. DYNHYD is a hydrodynamic procedure, DYNHYD procedure with exercise equation and continuous equation[8] for foundation. WPSP imitates the sport and interaction of various pollutant in water. The way (x|the y right angle sit to mark a broken line graph) that the WASP fluid matter model can output a result through a data form as well as pass sketch keeps a view to show the result that model circulates. WASP imitates procedure to constitute to $^{\circ}$ from two statures procedures: poisonous chemistry thing model TOXI and rich nourishment turn model EUTRO .

TOXI mold piece: It can predict to fuse Tai and adsorb Tai chemistry the thing is in the variety circumstance in the river. The pollution of TOXI mold piece emulation poisonous material can consider 1 3 kinds of chemistry materials and 1 3 kinds of grain materials, including organic compound, metal and sediment etc. Can compute respectively to some pollution material, it fuses Tai and the density of grain Tai in water body, in the bottom mire hole water and the solid bottom density within mire[9]. 


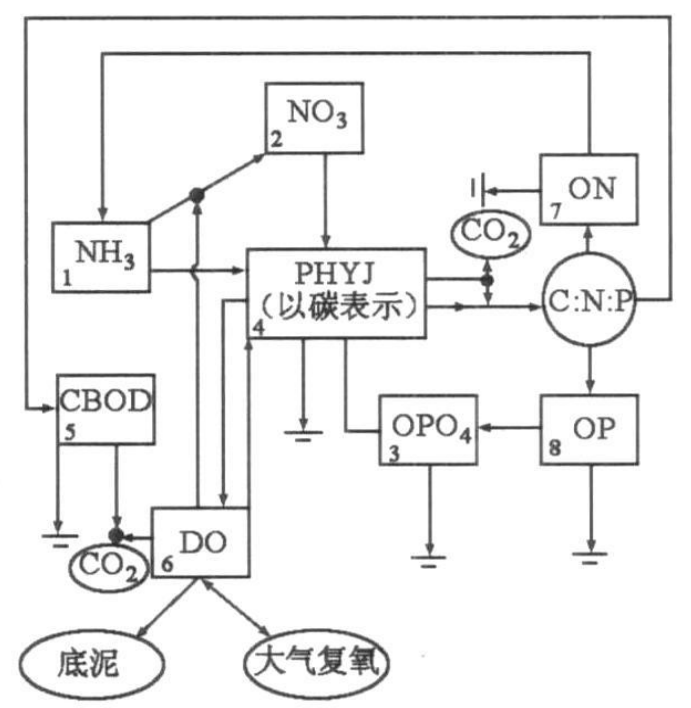

Figure 1. Relationships between WASP eutrophication state variables

EUTRO mold piece: Adopt rich nourishment of POTOMAC to turn the dynamics of model to combine WASP to move structure, the model imitated 8 normal regulations fluid matters index sign, can immediately predict $\mathrm{DO}, \mathrm{COD}, \mathrm{BOD}$, rich the nourishment turn, carbon, chlorophyll a, ammonia, nitrate, organic nitrogen, positive phosphoric acid salt etc. material is in the variety circumstance in the river[10].See Fig.1.

\section{THE APPLICATION OF WASP MODEL}

The operation method of WASP model is a river net first model to all turn, then carry on according to as follows 4 main steps: Water power studies, the quality deliver a research, fluid matter conversion research and environment poison the reason learn a research .Square one water power research wants to apply water power model procedure DYNHYD; Delivering of material in the second step research water current, depend show a drug research and the TOXI mold piece school of the fluid matter model procedure WASP check to complete; The third step research water current and the material conversion in the bottom quality, depend on laboratory research, the spot observation and experiment, parameter estimate, the model research combine together to complete, its model computes as a result want to verify; The end one step studies pollutant how influence environment[11].

\section{APPLIES WASP SOFTWARE[12]}

The WASP software works window way one behavior the bottom pull menu, there is File, Pre-processor, Model, Post-processor, Help. The second behavior fast function presses button.

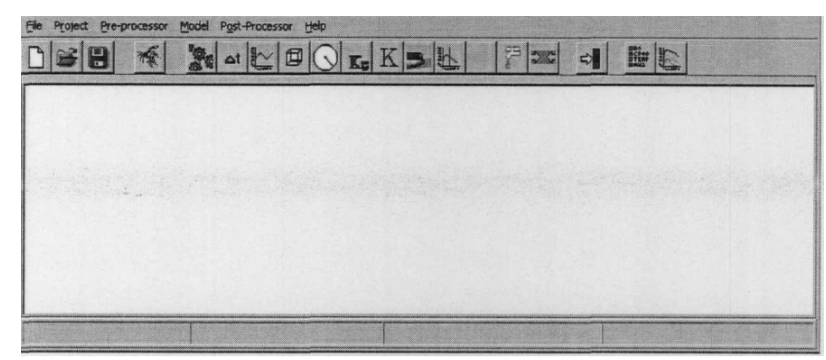

\section{A. Input the establishment of document}

Choose New in the WASP system main course list File, the options built up a new importation document. Pass main course after inputing a document establishment list Pre one Processor of next pull menu to carry out logarithms according to of importation and editor.

\section{B. The data inputs}

Water body physical volume, each parameters, such as length, width and depth...etc. presses the request of WASP software and pursue an item input one Processor to Pre of next pull to make a list 1 in the medium Segment, is shown as diagram.



\section{The parameter inputs}

The parameter wanting to input can classify 4 types of: environment parameter (include to imitate type RUTRO or TOXI), deliver parameter, boundary parameter and conversion parameter.

\section{The importation of discharge}

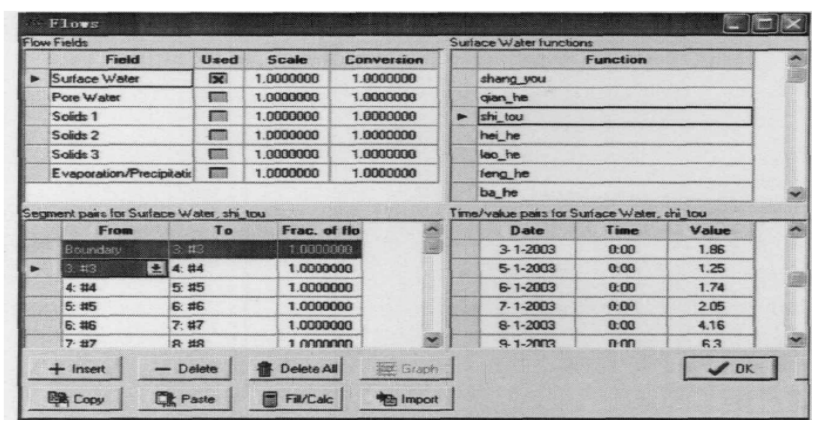




\section{E. The pollutant parameter inputs}

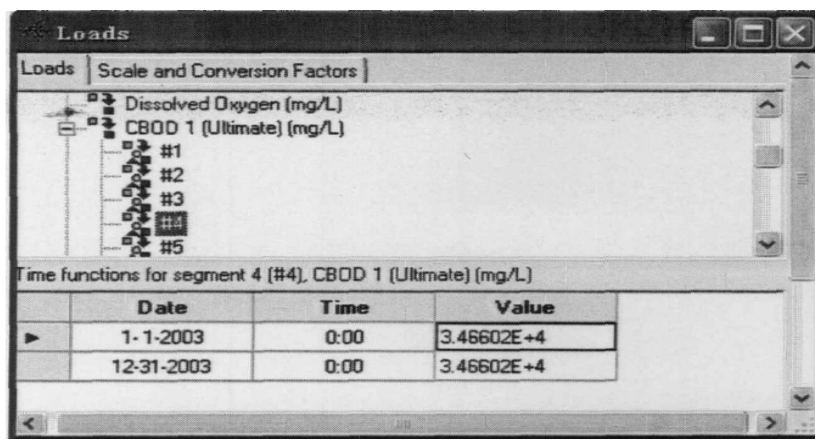

F. The performance of model

Model once acquiring a complete importation data, can start emulation, the window way will show each river segment, each change to measure the emulation in each time result. The network structure of the length and emulation river segment that imitates a process demand for time and change to measure of how much relevant.

\section{WASP IS APPLIED}

Since the 80 's in 20th century, WASP model puts forward. Abroad, Thomann and Fitzpatrick give fluvial rich nourishment of horse to turn to carry on emulation to wave in the east of the United States; The volatility organic matter pollution of Ambrose to the east of the United States Delaware port carries on emulation. In China, Pang-Yong etc. [13] carried algae on Tai Lake to imitate a research and inquired into Tai Lake in the lake variety mechanism, to manage too there is certain realistic meaning in "algae"; Liao Zhen-Liang ect. in Tong-ji university wait[14] WASP models carried on two developments and built up river fluid matter model in Suzhou, and make use of the model synthesizes to river environment in Suzhou whole cure engineering in relevant engineering and project carried on an emulation calculation; Yang Jia-Kuan waits[15] make use of WASP6 estimate south water north adjust behind the fluid matter of the segment of Xiang Fan, end of the movement result make people all more satisfied. DO in the Dong-Chang lake imitates a value and actually measures value of on the average opposite error margin only is 4.16\%[16].See Fig 2 .

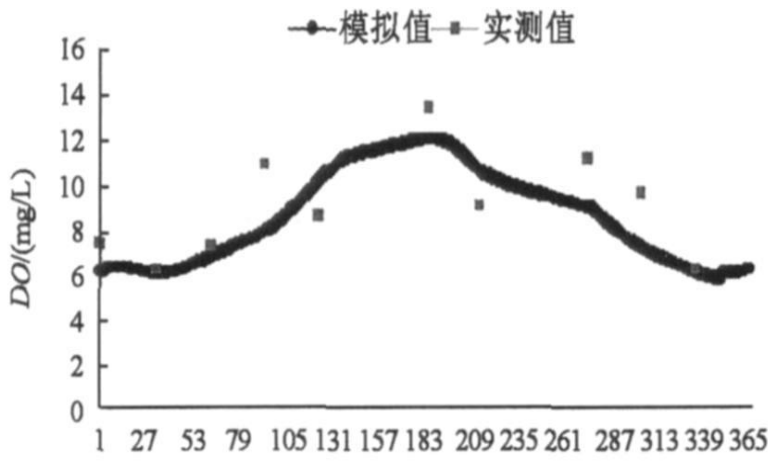

$t / \mathrm{d}$
Figure 2. Error comparison between the simulated value and the measured value of DO

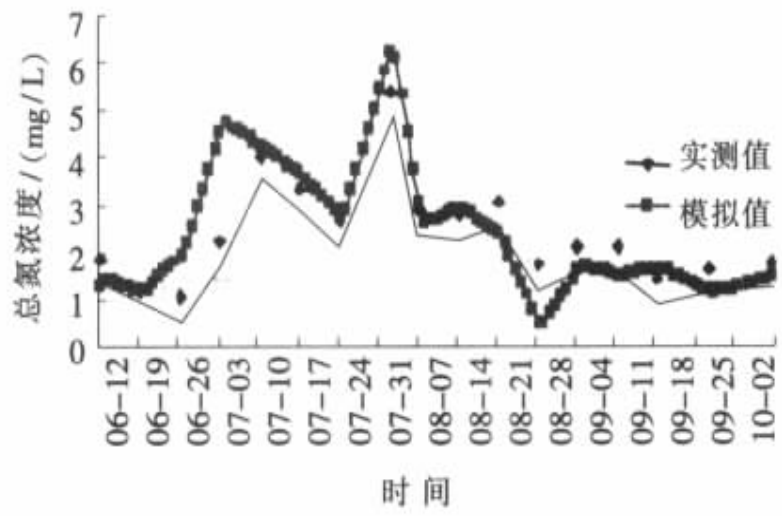

Figure 3. Error comparison between the simulated value and the measured value of TN

The piece is in the Zhang Yong-Xiang 《according to the application study of WASP7.2 river fluid matter model 》 apply an EUTRO mold piece within WASP model to carry on emulation to long river water fluid matter inside Peking City. Express that imitating a value and actually measuring to be worth an error margin is smaller as a result, number curve the relativity is better, basic request of emulation for matching model. The model can distinguish to carry on emulation to the COD in the fluid matter index sign and TN, TP and chlorophyll. Underneath only the TN actually measure value and model to compute an achievement to carry on a comparison. The TN index sign imitates values and actually measure the comparison diagram of value detailed see figure 3[17].Apply the solid example fluid matter emulation that all expresses WASP at home and abroad can a little bit well imitate various fluid matter process.

\section{SUMMARIES}

Pass to apply a solid example at home and abroad, the analysis actually measures value and emulation to be worth more curvilinear variety trend is very approach, basic request of emulation for matching model. Prove the model to have bigger rationality and credibility.

\section{REFERENCES}

[1] Luos are certainly expensive, Wang Xue Jun, rather, Sun Li waits. The fluid matter model research progress and river valley manage the model WARMF comment[J].Water science makes progress, 2005,(2):289-294.

[2] Di Toro DM.Sifitzpartic JJ.Documentation for Water Quality Program ( WASP) and Model Verification Program(MVP) [Z].Duluth ,MN:US Environmental Protection Agency ,1983.

[3] Ambrose RB , Wool TA, Connolly J P. WASP4 , A Hydro2dynamic and Water Quality Model - model Theory, User's Manual and Programmer's Guide[Z].Athens, GA: US Environmental Protection Agency ,1988.

[4] Ambrose RB , Wool TA, Martin JL, et al. WASP5, A Hy2drodynamic and Water Quality Model - model Theory, User's 
Manual and Programmer's Guide [Z].Draft : , GA:Environmental Reasearch Laboratory, US EnvironmentalProtection Agency, 1993.

[5] TimA. Wool, RobertB. Ambrose , JamesL. Martin , etal.Water Quality Analysis Simulation Program (WASP) Version 6. 0 DRAFT: User's Manual [Z].Atlanta : US Environmental Protection Agency, MS Tetre Tech. 2001.

[6] slearn, Deng Xiao Long, Zhang Cai Xiang, wait.The WASP6 systemses imitate really in the database area fluid matter in Yangtse gorges in of application[J].University college journal in Yangtse gorges, 2003,(2): 185

[7] breadth in the house, the Xiao wave, Liu Nian Feng, waits.The WASP6 fluid matter model is applied to man river Xiang Fan segment the fluid matter imitate a research[J]. Water resources protects, 2005 , :(4)8-10.

[8] Lius always, Guo keeps in mind, Fan Ying Ying, wait.The lake ecosystem dynamics model research makes progress[J].Applied ecosystem college journal, 2005,(6) :1169-1175.

[9] wears city Xian, the Yan has Lu.The applied TOXI model carries on the research that the heavy metals moves to change regulation in the river[J].The environmental science makes progress, 1995,(3):70-74.

[10] He Meng often, Yang Ju Ya Rong.Fluid matter model, ecosystem model and calculator model software[J].The environmental science makes progress, 1999,(3) :62-68

[11] Liaos flap good, Lin Wei Qing, Xu Zu Xin.Systems WASP-5 and it says to review[J].Environmental science in Shanghai, 2001,(1):3-6.

[12] Cheng yi-man, Study On water analysis for Weihe River in Shanxi Based on WASP7,Northwest University,master dissertation,2008

[13] Pangs are brave.Too the comprehensive number of atmosphere-water in the lake region environment studies[M].Peking:weather publisher, 1998.50-55.

[14] Liaos flap good, Xu Zu Xin, Gao Ting Yao.River environment in Suzhou synthesizes the whole model analysis that cure engineering fluid matter[J].Together help an university college journal, 2004,(4) :499-502.

[15] breadth in the house, the Xiao wave, Liu Nian Feng, waits.WASP6 estimate south water north adjust behind the fluid matter of the segment of Xiang Fan[J].Chinese water supply drains, 2005,(9) :103104.

[16] SUN Wen Zhang's paper:the of water quality research of Dongchang of Simulation Lake using WASP, [J].JOURNAL OF SHANDONG UNIVERSITY ( ENGINEERING SCIENCE).2008(4):83-85

[17] piece always Xiang etc., according to the application study of WASP7.2 river fluid matter model[J].Water in Peking works, 2010(1)31-33. 